\title{
Control over multiscale mixing in broadband-forced turbulence
}

\author{
Arkadiusz K. Kuczaj ${ }^{1}$ and Bernard J. Geurts ${ }^{1,2}$ \\ 1 Department of Applied Mathematics, J.M. Burgers Center for Fluid Mechanics, \\ University of Twente, P.O. Box 217, 7500 AE Enschede, The Netherlands \\ 2 Department of Applied Physics, Eindhoven University of Technology, \\ P.O. Box 513, 5600 MB Eindhoven, The Netherlands
}

The effects of explicit flow modulation on the dispersion of a passive scalar field are studied. Broadband forcing is applied to homogeneous isotropic turbulence to modulate the energy cascading and alter the kinetic energy spectrum. Consequently, a manipulation of turbulent flow can be achieved over an extended range of scales beyond the directly forced ones. This modifies transport processes and influences the physical-space turbulent mixing of a passive scalar field. We investigate by direct numerical simulation the stirringefficiency associated with turbulence modified by forcing. This is quantified by monitoring the surface-area and wrinkling of a level-set of the passive scalar field. We consider different forcing to manipulate the quality and rate of mixing. The instantaneous mixing efficiency measured in terms of surface-area or wrinkling is found to increase when additional energy is introduced at the smaller scales. The increased intensity of small scales significantly influences the small-scale mixing characteristics depicted by wrinkling, while the forcing of large scales primarily affects the surface-area. Evaluation of geometrical statistics in broadband-forced turbulence indicates that the self-amplification process of vorticity and strain is diminished. This leads generally to smaller extremal values of the velocity gradients but higher average values as a result of the competition between the natural cascading processes and the explicit small-scales forcing.

\section{Introduction}

Turbulent mixing of embedded scalar fields is important in a diverse range of fluid mechanics problems, from process-engineering, environmental issues to non-premixed combustion. The efficiency of mixing is governed by a number of aspects. Nowadays, growing computational capabilities allow the determination of statistics of turbulent flows at quite high Reynolds and Schmidt numbers [1]. Simultaneously, the engineering approach is directed towards control 
of mixing by modulation of the driving velocity fields [2]. Recently, the use of multiscale forcing methods was proposed to model turbulent flows that are disturbed at various spatial scales [3] as may arise in case of flows through complex geometrical structures such as metal foams, or forest canopies. These numerical experiments indicate that turbulent mixing properties, e.g., expressed by surface-area and surface-wrinkling growth-parameters of scalar level-sets, can be significantly influenced by external agitation.

We consider the incompressible Navier-Stokes equations with broadband forcing working as a complex stirrer in which a spectrum of length-scales is simultaneously perturbed. Traditionally, only large-scale forcing was included in a simulation. This induces an average flow of energy toward smaller scales. The additional forcing in a high wavenumber band agitates a specific range of spatial scales as depicted in Fig. 1(a). We focus on the control over basic mixing-properties that may be obtained from such explicit broadband forcing and concentrate on the consequences (i) for the time needed to reach a perfectly-mixed state and (ii) the accumulated large- and small-scale mixing.
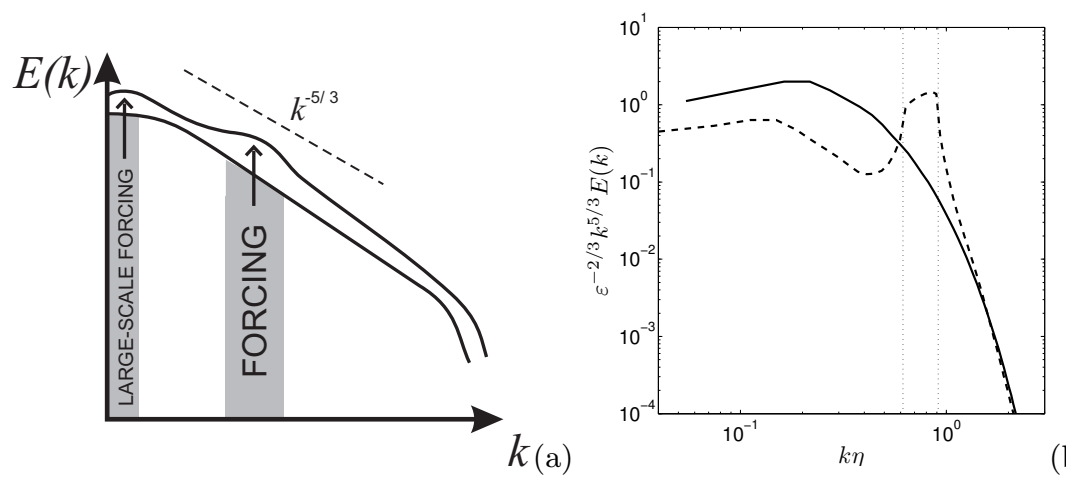

Fig. 1. (a) Broadband forcing in spectral space. (b) Time-averaged compensated energy spectrum for the large-scale $\left(\mathbb{K}_{1,1}\right.$ : solid) and additional broadband $\left(\mathbb{K}_{17,24}\right.$ : dashed) forced turbulence, where $\varepsilon$ is the energy-dissipation rate and $\eta$ - Kolmogorov scale.

We investigate the dispersion of strongly localized initial scalar concentrations. Direct numerical simulation of the forced turbulence shows that the maximal surface-area and wrinkling as well as the time at which such a maximum is achieved can be controlled by variation of forcing parameters. The time-integrated surface-area and wrinkling are indicators of the accumulated effect. The simulations show that at small Schmidt numbers, a higher emphasis on small-scale flow agitation yields a significant increase in the mixing. 


\section{Computational flow model}

We solve the incompressible Navier-Stokes equations in spectral space:

$$
\left(\partial_{t}+\operatorname{Re}^{-1} k^{2}\right) u_{\alpha}(\mathbf{k}, t)=M_{\alpha \beta \gamma} \sum_{\mathbf{p}+\mathbf{q}=\mathbf{k}} u_{\beta}(\mathbf{p}, t) u_{\gamma}(\mathbf{q}, t)+F_{\alpha}(\mathbf{k}, t),
$$

where $u_{\alpha}(\mathbf{k}, t)$ is the velocity field coefficient at wavevector $\mathbf{k}(k=|\mathbf{k}|)$ and time $t$, and Re is the computational Reynolds number. The tensor $M_{\alpha \beta \gamma}=$ $\left(k_{\beta} D_{\alpha \gamma}+k_{\gamma} D_{\alpha \beta}\right) /(2 \imath)$, where $D_{\alpha \beta}=\delta_{\alpha \beta}-k_{\alpha} k_{\beta} / k^{2}$, accounts for the pressure and incompressibility effects. We adopted a forcing procedure motivated by flow through a fractal gasket [4]. This particular forcing has a constant energy input rate $\varepsilon_{w}$ for the entire system:

$$
F_{\alpha}(\mathbf{k}, t)=\frac{\varepsilon_{w} k^{\beta}}{\sum_{\mathbf{k} \in \mathbb{K}_{m, p}} \sqrt{|\mathbf{u}(\mathbf{k}, t)|} k^{\beta}} e_{\alpha}(\mathbf{k}, t) .
$$

Each forced band $\mathbb{K}_{m, p}(m \leq p)$ consists of $p-m+1$ adjacent spherical shells $\mathbb{S}_{n}=2 \pi(n-1 / 2) / L_{b}<|\mathbf{k}| \leq 2 \pi(n+1 / 2) / L_{b}: m \leq n \leq p$. Here $L_{b}$ is the size of the computational domain. We force the first shell $\mathbb{K}_{1,1}$ with a constant energy injection rate $\varepsilon_{w, 1}$ and a single high- $k$ band $\mathbb{K}_{m, p}$ with $\varepsilon_{w, 2}$. The vector $\mathbf{e}(\mathbf{k}, t)=\mathbf{u}(\mathbf{k}, t) /|\mathbf{u}(\mathbf{k}, t)|+\imath \mathbf{k} \times \mathbf{u}(\mathbf{k}, t) /(|\mathbf{k}||\mathbf{u}(\mathbf{k}, t)|)$ has the general form proposed in [4] and the complexity of the stirring object is parameterized by the exponent $\beta=D_{f}-2$ related to the fractal dimension $D_{f}$.

The scalar concentration $C$ evolves in a velocity field $\mathbf{v}$ by:

$$
\partial_{t} C(\mathbf{x}, t)+(\mathbf{v}(\mathbf{x}, t) \cdot \nabla) C(\mathbf{x}, t)=(\operatorname{ReSc})^{-1} \nabla^{2} C(\mathbf{x}, t),
$$

where Sc is the Schmidt number. We adopt a level-set integration method to quantify basic mixing-properties of the evolving scalar fields [5]. Geometric properties of a level-set $S(a, t)=\left\{\mathbf{x} \in \mathbb{R}^{3} \mid C(\mathbf{x}, t)=a\right\}$ may be evaluated by integrating a corresponding 'density function' $g$ over this set. In fact, we have:

$$
I_{g}(a, t)=\int_{S(a, t)} d A g(\mathbf{x}, t)=\int_{V} d \mathbf{x} \delta(C(\mathbf{x}, t)-a)|\nabla C(\mathbf{x}, t)| g(\mathbf{x}, t)
$$

where the volume $V$ is the flow-domain. Setting $g(\mathbf{x}, t)=1$ we may determine the surface-area $A$ of $S$. In case $g(\mathbf{x}, t)=|\nabla \cdot \mathbf{n}(\mathbf{x}, t)|$, where $\mathbf{n}(\mathbf{x}, t)=$ $\nabla C(\mathbf{x}, t) /|\nabla C(\mathbf{x}, t)|$ is a unit normal vector, we can determine the wrinkling $W$ of $S$. We focus on the evolution of the surface-area and wrinkling, monitoring the instantaneous value as well as the accumulated effect:

$$
\vartheta_{Z}(a, t)=\frac{I_{Z}(a, t)}{I_{Z}(a, 0)} ; \zeta_{Z}(a, t)=\int_{0}^{t} \vartheta_{Z}(a, \tau) d \tau ; Z \in\{A, W\}
$$

By determining $\vartheta_{A}$ and $\vartheta_{W}$ we may quantify the rate at which surfacearea and wrinkling develop, the maximal values that are obtained and the time-scale at which these are achieved. The cumulative measures $\zeta_{A}(a, t)$ and $\zeta_{W}(a, t)$ express the total surface-area and wrinkling that has developed in the course of time. 


\section{Mixing efficiency}

The broadband forcing is observed to modify the kinetic energy spectrum in a strongly non-local manner as seen in Fig. 1(b). This illustrates the deviations from the classical Kolmogorov picture that is characterized by a $-5 / 3$ slope in the spectral energy distribution (for more details see [3]).
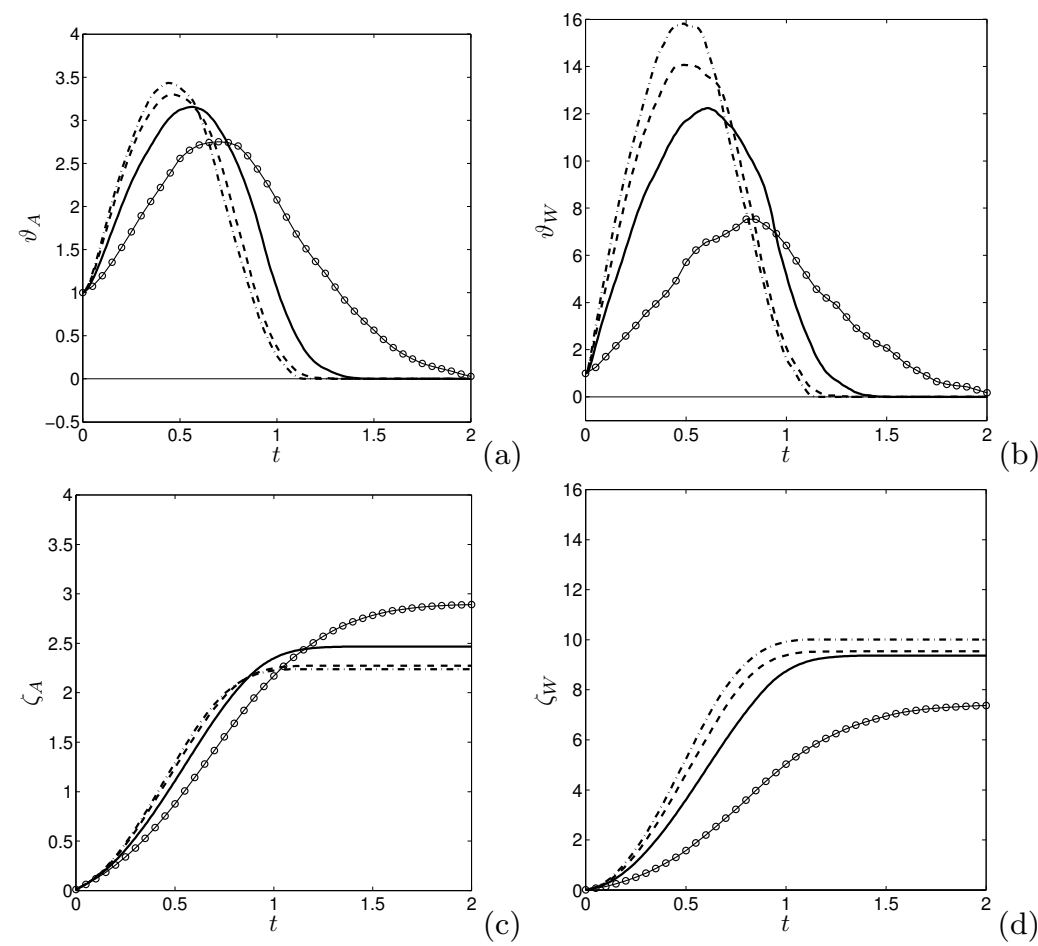

Fig. 2. Evolution of passive scalar dispersion parameters: a) surface-area $\vartheta_{A}$, b) wrinkling $\vartheta_{W}$, c) accumulated surface-area $\zeta_{A}$, d) accumulated wrinkling $\zeta_{W}$. Large-scale forcing $\mathbb{K}_{1,1}$ with $\varepsilon_{w}=0.15$ and additional forcing in the band $\mathbb{K}_{5,8}$ at $\varepsilon_{w, 2}=0,0.30,0.45,0.60$ (o, solid, dash, dash-dotted).

To establish the influence of forcing on mixing properties we simulated the spreading of a passive tracer at Schmidt number $S c=0.7$ with turbulence at $R_{\lambda} \approx 50(R e=1061)$. The simulations started from a spherical tracer distribution $C$ of radius $r=3 / 16$ scaled to be between 0 and 1 and the level-set $a=1 / 4$ was considered. The resolution requirements were satisfactorily fulfilled: $k_{\max } \eta$ ranges from 2.3 to 3.5 using a resolution in the range $128^{3}-192^{3}$ grid-cells. Here $k_{\max }$ is the highest wave-number that is resolved in the simulation. For the passive scalar these resolutions correspond to $k_{\max } \eta_{O C}$ in the 
range from 3 to 4.5 , where $\eta_{O C}$ is the Obukhov-Corrsin scale [6]. The characterization of the mixing-efficiency was based on an ensemble of 20 simulations, each starting from an independent fully-developed realization of the velocity field. Individual velocity fields were separated by two eddy-turnover times.

In Fig. 2 we compare the instantaneous and accumulated mixing properties for a number of forcing parameters. We include large-scale forcing in $\mathbb{K}_{1,1}$ at $\varepsilon_{w, 1}=0.15$ as well as forcing of the band $\mathbb{K}_{5,8}$ at various $\varepsilon_{w, 2}$. We observe that the large-scale forcing mainly governs the development of the surfacearea, while forcing in the second band has a larger influence on the wrinkling. An increase in the strength of the forcing in $\mathbb{K}_{5,8}$ leads to a slight increase in $\vartheta_{A}$ and a considerable reduction in the time at which $\vartheta_{A}$ reaches its maximum. The final cumulative surface area, however, decreases with increasing $\varepsilon_{w, 2}$. In contrast, an increase in $\varepsilon_{w, 2}$ quite strongly influences the instantaneous wrinkling; the maximal value increases and the time of maximal mixing decreases. The cumulative effect on $W$ increases notably with increasing $\varepsilon_{w, 2}$. Similar results were obtained when forcing the band $\mathbb{K}_{13,16}$ instead.
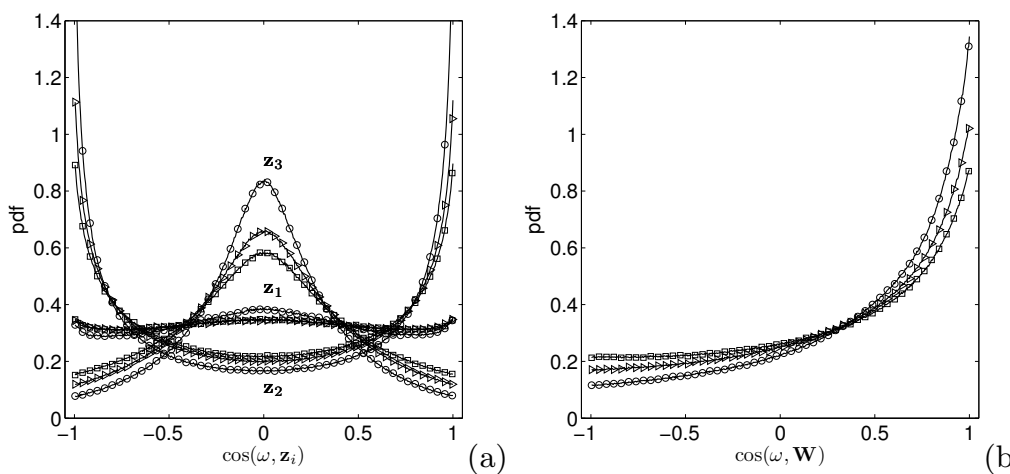

Fig. 3. PDFs of the cosine of the angle between vorticity $\boldsymbol{\omega}$ and (a) the eigenvectors $\mathbf{z}_{i}$ of the rate of strain tensor, and (b) the vortex stretching vector $\mathbf{W}$, for largescale forcing $\mathbb{K}_{1,1}$ at $\varepsilon_{w, 1}=0.15$ and additional broadband forcing with energy input $\varepsilon_{w, 2}=0,0.15,0.45$ to the second band $\mathbb{K}_{17,24}(\circ, \triangle, \square)$.

Evaluation of geometrical statistics [7] shows that broadband forcing considerably changes the general characteristics of turbulence. The selfamplification process of vorticity and strain is diminished and the statistical flow-structure altered. This can be seen in Fig. 3 where the pdfs of the alignment between the vorticity $\boldsymbol{\omega}=\nabla \times \mathbf{u}$ and eigenvectors of the rate of strain and the vortex stretching vector are plotted for various forcing-strengths in the second band. Increased forcing of the small scales leads to less pronounced alignment. 


\section{Conclusions}

Forcing methods agitating a flow in a wide range of scales induce significant differences compared to the case obtained classically in which only the large scales are forced. In this study we devoted attention to a recently proposed multiscale forcing that models a flow under the influence of an additional perturbation by a complex stirrer [4]. We performed numerical simulations of the dispersion of a passive scalar field in a turbulent flow that is driven by such forcing.

By monitoring global properties of level-sets of the evolving passive scalar we could quantify the modification of the mixing that results from broadband forcing. It was found that broadband forcing causes additional production of smaller scales in the flow. This is directly responsible for the enhancement of wrinkling. In contrast, the surface-area of a level-set of the tracer is found to be mainly governed by convective sweeping by the larger scales in the flow. Hence, the surface-area is controlled to a greater extent by the energy injected at the largest scales.

The additional energy introduced by forcing at small scales competes with processes that govern Kolmogorov-type turbulence, e.g., expressed by the selfamplification of vorticity and strain and vortex stretching. The forcing also changes the structure of turbulence; it modifies the alignment of vorticity with eigenvectors of the rate of strain tensor. Future study will be devoted to clarifying this role of the small-scale forcing by evaluating the geometrical statistics of the turbulent flow as function of forcing parameters.

\section{Acknowledgments}

This work is part of the FOM research program on turbulent flow. AKK would like to thank Arkady Tsinober (Imperial College, London) for many fruitful comments regarding geometrical statistics in turbulence.

\section{References}

1. P.K. Yeung, D.A. Donzis, and K.R. Sreenivasan. High-Reynolds-number simulation of turbulent mixing. PoF, 17:081703, 2005.

2. M. Kearney. Engineered fractal cascades for fluid control applications. In Proc. of Fractals in Engineering Conference, Arcachon, France. INRIA, June, 1997.

3. A.K. Kuczaj and B.J. Geurts. Mixing in manipulated turbulence. JoT, to appear.

4. B. Mazzi and J.C. Vassilicos. Fractal generated turbulence. JFM, 502:65, 2004.

5. B.J. Geurts. Mixing efficiency in turbulent shear layers. JoT, 2:2, 2001.

6. T. Watanabe and T. Gotoh. Statistics of a passive scalar in homogeneous turbulence. New J. Phys., 6(40):1, 2004.

7. A. Tsinober and B. Galanti. Exploratory numerical experiments on the differences between genuine and "passive" turbulence. PoF, 15(11):3514-3531, 2003. 